\title{
Prevalencia de helmintos enteroparásitos zoonóticos y factores asociados en Canis familiaris en una zona urbana de la ciudad de Ica, Perú
}

\author{
MARÍA DEL PILAR TRILLO-ALTAMIRANO*, ADELA JANNET CARRASCO*, y RUFINO CABRERA*
}

\section{PREVALENCE OF ZOONOTIC ENTEROPARASITE HELMINTHS AND ASSOCIATED FACTORS IN Canis familiaris IN AN URBAN AREA OF ICA CITY, PERU}

The aim of the investigation was to determine the prevalence of infection with enteroparasite helminths and to identify some associated factors in Canis familiaris, "dog» in an urban area of Ica city, in Southern Peru. A cross-sectional study was carried out between November and December 2001 in a number of 162 family dogs (pets) of both sexes, different ages and races, selected by a bistage sampling. Two samples per animal were evaluated by means of direct exam and concentration (Faust and spontaneous sedimentation in test tubes). The general prevalence was $40.12 \%$, for Toxocara canis $19.75 \%$, Ancylostoma caninum 9.26\%, Dipylidium caninum $8.64 \%$, Toxascaris leonina $6.17 \%$, and Taenia sp. $4.32 \%$. The sex is not associated to infection for intestinal helminths, being younger than one year $p=0.00000002$, OR 9.74 IC 95\% (3.75 - 25.72) is the only potential risk factor that has been found for the infection with $\boldsymbol{T}$. canis.

Key words: Intestinal helminths, Canis familiaris, prevalence, Peru.

\section{INTRODUCCIÓN}

En caninos, los helmintos intestinales tienen importancia porque algunos como el Toxocara canis, causan anorexia, disminuyen el apetito y en infecciones masivas en los cachorros les pueden llevar hasta la muerte, y otros como Dipylidium caninum y Echinococcus granulosus interfieren en la absorción y conversión de nutrientes causando diarrea y obstrucción intestinal en casos de infección masiva ${ }^{1-2}$.

El perro juega un papel importante en la transmisión de infecciones helmínticas de tipo zoonóticas al hombre, como por ejemplo de la hidatidosis, grave enfermedad producida por los estados larvales de E. granulosus ${ }^{3}$. Así también el $T$. canis, Toxocara cati y Toxascaris leonina pueden producir en el hombre los síndromes clínicos denominados larva migrans visceral y ocular $^{1-2}$, que se adquieren por el consumo de huevos larvados de estos parásitos que contaminan el suelo de parques, jardines y otras áreas recreativas. También se puede producir el llamado larva migrans cutáneo en el hombre al

\footnotetext{
Facultad de Ciencias, Universidad Nacional San Luis Gonzaga de Ica, Perú. Casilla Postal 03. Ica, Perú. María Del Pilar Trillo-Altamirano E-mail: madpilar@ hotmail.com Rufino Cabrera E-mail: rcabrerach@ hotmail.com Adela Jannet Carrasco T E-mail: jannet09@ hotmail.com
} 
penetrar larvas de ancylostomideos del perro por su piel ${ }^{2,5}$. A pesar que la toxocarosis como problema en el hombre recién se esta reconociendo ${ }^{6}$, es necesario conocer la magnitud de las infecciones por los helmintos zoonóticos del perro en ciudades en donde no se tiene estos datos así como identificar los factores asociados a la infección animal que pueden servir para diseñar un programa de control.

Los resultados obtenidos en estudios similares en Latinoamérica son muy variables y es así como en Chile se han encontrado prevalencias entre 4,5 a $51,9 \%^{7-8}$, en Brasil entre 0,7 a 23,6\% ${ }^{9}$, Argentina de 5 a $18 \%{ }^{10}$ y México de 0,7 a 37,3\% ${ }^{11}$. En el Perú, la mayoría de estudios se han realizado en Lima $^{12-14}$, siendo escasos los realizados en otros departamentos ${ }^{15-17}$. En Ica, sólo existe un estudio sobre toxocarosis ${ }^{18}$ y otro sobre equinococosis en una zona del camal municipal en la ciudad de Chincha ${ }^{19}$.

El objetivo del presente trabajo fue determinar y analizar las prevalencias de infección de las enteroparasitosis del perro en la ciudad de Ica, al sur del Perú.

\section{MATERIAL Y MÉTODOS}

El estudio se realizó en la localidad denominada La Palma Grande, zona urbana ubicada en el cercado de la ciudad de Ica, provincia y departamento del mismo nombre al sur de Lima, sus coordenadas geográficas son $13^{\circ} 01$ ' $15^{\prime \prime}$ Latitud Sur y 76 24'26" Latitud Oeste.

La población en estudio fue de 642 perros cifra que fue obtenida a través de un censo. El tamaño de la muestra se calculó mediante el software Epi Info 6.04, se consideró una prevalencia hipotética de $15 \%$ y un margen de error de $\pm 0,05$ y un nivel confianza al $95 \%$, además, se agregó como margen de seguridad el $8 \%$ a la muestra mínima lo que significó trabajar 162 caninos.

Se incluyeron en el estudio a perros con dueño mayores de 3 meses de edad, de ambos sexos, de las raza mestiza, Siberian Husky, Pekinés, Cocker Spaniel, Ovejero, Samoyedo, Doberman, Chow-Chow, Boxer, Bulldog y Labrador que habitaban en la Palma Grande y que no habían recibido tratamiento antihelmíntico en los últimos 15 días antes de la toma de muestra. Los animales se seleccionaron mediante un muestreo bietápico, para seleccionar la vivienda (unidad primaria de muestreo) se utilizó el muestreo sistemático y para seleccionar el animal en la vivienda (unidad secundaria de muestreo) se utilizó un muestreo aleatorio. En todos los casos se solicitó la autorización de los dueños para incluir sus mascotas en el estudio. Cuando los dueños no aceptaron que sus animales ingresen al estudio, fue reemplazado por otro más cercano.

Se diseñó un estudio descriptivo - transversal porque se evaluó la prevalencia lápsica y se describe por las variables sexo y edad, además, se evaluó si estas variables están asociadas a la infección por vermes enteroparásitos. Se definió como caso a los animales que resultaron positivos a helmintos al examen coproparasitológico.

\section{Procedimientos y métodos de diagnóstico:}

Se recolectaron por las mañanas aproximadamente $15 \mathrm{~g}$ de heces frescas en dos días diferentes por cada animal en envases herméticos. En el caso de que no se analizaran el mismo día se fijaron en formol al $10 \%$. Se procesaron simultáneamente por el método directo (solución salina fisiológica y lugol), la concentración de Faust ${ }^{20}$ y Sedimentación Espontánea en Tubo de Ensayo $(\mathrm{SET})^{21}$. La identificación de los parásitos se basó en sus características morfológicas y en las mediciones biométricas de sus huevos en un microscopio Kyowa Optical LSSB-VC-2 con un ocular micrométrico calibrado y comparación con las descripciones en los textos $2,3,20,22$.

Para determinar la prevalencia se utilizó la frecuencia porcentual simple, el Chi-q de independencia y para identificar los factores de riesgo potenciales se utilizó la prueba de diferencia de proporciones " $p$ " y el odds ratio (OR) que se calculó en una tabla de 2 x 2 utilizando un intervalo de confianza (IC) al 95\% en Epi Info 6.04.

\section{RESULTADOS}

De 162 perros examinados, $65(40,12 \%)$ presentaron uno o más especies de helmintos. La prevalencia en machos fue $20,37 \%$ y en hembras $19,75 \%$. Estas diferencias no fueron significativas $(\mathrm{p}=0,3996)$.

En la Tabla 1 se presenta la prevalencia de acuerdo a las especies de helmintos. Entre los céstodes el $D$. caninum $(8,64 \%)$ fue el más frecuente, seguido por Taenia sp. (4,32\%) y entre los nemátodos $T$. canis $(19,75 \%)$, seguido de $A$. caninum $(9,26 \%)$ y $T$. leonina $(6,17 \%)$. 
En la Tabla 2, se presenta las asociaciones parasitarias. La más frecuente fue el monoparasitismo $(83,07 \%)$, la especie predominante fue T. canis $(36,92 \%)$, seguido del biparasitismo $(13,85 \%)$ por $T$. canis + D. caninum $(7,69 \%)$.

En la tabla 3 se presenta los resultados del sexo y la edad como posibles factores asociados a la infección por helmintos enteroparásitos. Existe diferencia estadísticamente significativa en la prevalencia entre machos y hembras por $A$. caninum, pero el intervalo mínimo del OR es 0,91. De acuerdo a la edad se encontró que entre los infectados por T. canis hay 10 veces más riesgo de estarlo, en los menores de 1 año OR 9,74 IC $95 \%(3,75-25,72), \mathrm{p}=0,00000002$.

\section{DISCUSIÓN}

La prevalencia de helmintos intestinales en la población canina en una zona urbana de la ciudad de Ica es inferior al reportado por métodos parasitológicos ${ }^{13}$, lo que se explica por el tipo de población evaluada. Los perros examinamos en el presente estudio son de casa a diferencia del trabajo anterior que evaluó animales sin dueño (callejeros) que tendrían mayor riesgo de infectarse, mediante necropsia o la administración de vermífugos se han hallado prevalencias más elevadas en otros lugares del país ${ }^{15,16}$.

Al analizar la prevalencia por especies de helmintos enteroparásitos, nuestros resultados para $T$. canis son superiores a los encontrados en dos estudios previos ${ }^{13,14}$. Mediante el método de necropsia se han hallado valores más elevados
Tabla 1. Prevalencia por helmintos enteroparásitos en Canis familiaris en la urbanización La Palma, Ica, Perú. Septiembre-Noviembre 2001

\begin{tabular}{lrr}
\hline Especies & Positivos & Prevalencia (\%) \\
\hline Toxacara canis & 32 & 19,75 \\
Ancylostoma caninum & 15 & 9,26 \\
Dipylidium caninum & 14 & 8,64 \\
Toxascaris leonina & 10 & 6,17 \\
Taenia sp. & 7 & 4,32 \\
\hline
\end{tabular}

que el nuestro en Huancayo, Junín ${ }^{16}$ y en Chincha (Departamento de Ica) ${ }^{18}$, atribuible al método de diagnóstico, número de muestras, edad y a las condiciones ecológicas disímiles.

Al comparar nuestro resultado con autores Latinoamericanos, la prevalencia aquí encontrada es superior a la obtenida en zonas urbanas de Chile $^{8}$, Argentina ${ }^{10}$ y São Paulo, Brasil ${ }^{9}$.

Los resultados obtenidos en el presente estudio indican que la zona urbana de Ica debe ser considerada como de riesgo potencial para adquirir toxocarosis. En Perú, ya se han descrito 3 casos de la forma visceral en niños de $\operatorname{Lima}^{6}$ y 21 casos de la forma ocular ${ }^{23}$, la mayoría con antecedentes de crianza de perros y gatos, casi la mitad son niños, que estarían en mayor riesgo debido al mayor contacto con los perros. Por examen serológico se ha reportado $7,33 \%$ en sujetos de Lima ${ }^{24}$. Es posible que estudios similares en otras zonas del país, entre ellos en Ica, indiquen resultados similares.

La prevalencia hallada para A. caninum en

Tabla 2. Asociación parasitaria por helmintos enteroparásitos en Canis familiaris en la urbanización La Palma, Ica, Perú. Septiembre-Noviembre 2001

\begin{tabular}{lcc}
\hline Asociación & $\mathbf{N}^{\mathbf{0}}$ & Frecuencia $(\boldsymbol{\%})$ \\
\hline Monoparasitismo: & & 36,92 \\
Toxocara canis & 24 & 18,46 \\
Ancylostoma caninum & 12 & 9,23 \\
Toxascaris leonina & 6 & 9,23 \\
Dipylidium caninum & 6 & 9,23 \\
Taenia sp. & 6 & 7,69 \\
Biparasitismo: & 5 & 1,54 \\
Toxacara canis + Dipylidium caninum & 1,54 \\
Dipylidium caninum + Ancylostoma caninum & 1 & 1,54 \\
Toxocara canis + Ancylostoma caninum & 1 & 1,54 \\
Toxascaris leonina + Taenia sp. & 1 & 1,54 \\
Toxascaris leonina + Ancylostoma caninum & & \\
Triparasitismo: & 1 & \\
Dipylidium caninum + Toxacara canis + Toxascaris leonina & & \\
\hline
\end{tabular}


Tabla 3. Factores asociados a helmintos enteroparásitos en Canis familiaris en la urbanización La Palma, Ica, Perú. Septiembre-Noviembre 2001

\begin{tabular}{|c|c|c|c|c|c|}
\hline Exposición & & Especie & Valor de "p" & OR & IC $95 \%$ \\
\hline & & Toxocara canis & & & \\
\hline Sexo: & Infectado & No infectado & & & \\
\hline Hembra & 14 & 72 & 0,92989300 & 0,97 & $0,41-2,27$ \\
\hline Macho & 18 & 58 & & & \\
\hline \multicolumn{6}{|l|}{ Edad: } \\
\hline$<1$ Año & 20 & 19 & 0.00000002 & 9,74 & $3,75-25,72$ \\
\hline >1 Año & 12 & 111 & & & \\
\hline \multicolumn{6}{|c|}{ Ancylostoma caninum } \\
\hline Sexo: & Infectado & No infectado & & & \\
\hline Hembra & 11 & 61 & 0,03991661 & 3,07 & $0,91-10,86$ \\
\hline Macho & 5 & 85 & & & \\
\hline \multicolumn{6}{|l|}{ Edad: } \\
\hline$<1$ Año & 1 & 38 & 0,07991293 & 0,19 & $0,01-1,47$ \\
\hline >1 Año & 15 & 108 & & & \\
\hline \multicolumn{6}{|c|}{ Dipylidium caninum } \\
\hline Sexo: & Infectado & No infectado & & & \\
\hline Hembra & 9 & 63 & 0,20449180 & 2,00 & $0,60-6,80$ \\
\hline Macho & 6 & 85 & & & \\
\hline \multicolumn{6}{|l|}{ Edad: } \\
\hline$<1$ Año & 4 & 35 & 0,80584475 & 1,16 & $0,29-4,36$ \\
\hline$>1$ Año & 11 & 112 & & & \\
\hline \multicolumn{6}{|c|}{ Toxascaris leonina } \\
\hline Sexo: & Infectado & No infectado & & & \\
\hline Hembra & 5 & 67 & 0,71595515 & 1,27 & $0,30-5,38$ \\
\hline Macho & 5 & 85 & & & \\
\hline \multicolumn{6}{|l|}{ Edad: } \\
\hline$<1$ Año & 3 & 36 & 0,65191397 & 1,38 & $0,26-6,45$ \\
\hline$>1$ Año & 7 & 116 & & & \\
\hline Sexo: & Infectado & $\begin{array}{r}\text { Taenia sp. } \\
\text { No infectado }\end{array}$ & & & \\
\hline Hembra & 3 & 69 & 0,93135856 & 0,93 & $0,16-5,23$ \\
\hline Macho & 4 & 86 & & & \\
\hline \multicolumn{6}{|l|}{ Edad: } \\
\hline$<1$ Año & 1 & 38 & 0,53699945 & 0,51 & $0,02-4,61$ \\
\hline >1 Año & 6 & 117 & & & \\
\hline
\end{tabular}

este estudio es cercano a los reportados previamente en Lima $^{12-13}$, aunque no es comparable actualmente porque se asume que la prevalencia ha variado en las últimas 4 décadas debido a la mejora en las condiciones de sanidad animal; sin embargo, nuestro hallazgo es mucho menor al de Huancayo ${ }^{16}$.

En el Perú, se han reportado casos de larva migrans cutánea en la Selva y en la Costa de Lima $^{25}$, pero no se han establecido los agentes etiológicos. Los de la Costa de Lima probablemente han sido causados por A. caninum, aunque este parásito prevalece en zonas húmedas. La prevalencia elevada hallada podría explicarse por la migración de los dueños junto a sus mascotas y la presencia de factores microclimáticos que favorecen su enzootia.

La prevalencia hallada para $D$. caninum es cercano al de un estudio previo ${ }^{15}$, pero inferior a otros $^{13-14,16}$, disimilitudes explicables por el área de residencia, estrato socioeconónomico de sus dueños y método de diagnóstico. En algunas zonas urbanas de México ${ }^{11}$, Brasil ${ }^{9}$ y Argentina ${ }^{10}$ la tasa de prevalencia no es superior al $1 \%$; pero en 
localidades puntuales como en Ñuble, Chile fue $51,90 \%{ }^{7}$. La magnitud actual de la infección humana en el Perú no se conoce, pero se ha reportado una prevalencia de $0,5 \%$ en población de consulta externa en un hospital de Chincha ${ }^{26}$.

Nuestro resultado para T. leonina, es similar a un reporte ${ }^{15}$, pero inferior al comunicado por examen parasitológico ${ }^{13}$ y por necropsia en Chincha ${ }^{18}$, explicable como en los casos anteriores, en México, Argentina y Chile su prevalencia es menor o igual a $3 \% \%^{8,10-11}$.

La prevalencia hallada para Taenia sp. es superior a un hallazgo previo ${ }^{13}$, en otro estudio fue $2 \%$ para $T$. hydatigena ${ }^{15}$ y entre $9 \%$ a $22 \%$ para 3 especies de Taenia ${ }^{16}$, debido a la imposibilidad de distinguir los huevos lo presentamos como Taenia sp. Se sospecha que podría estar presente las tenias grandes, aunque no se descarta a $E$. granulosus, ya que se sabe que esta presente en perros de los alrededores del camal municipal de Chincha ${ }^{19}$.

El monoparasitismo por $T$. canis fue el más frecuente, seguido del biparasitismo por $T$. canis asociado a $D$. caninum, explicable por la elevada producción de huevos de $T$. canis y su resistencia a temperaturas extremas y a compuestos químicos que le confiere mayor capacidad de diseminación en el suelo (geohelminto), por lo tanto, mayor riesgo de infección. El predominio de $D$. caninum sería por la abundancia de pulgas.

El sexo no está asociado a la infección por helmintos intestinales, excepto para $A$. caninum que fue estadísticamente significativo, pero el OR fue marginalmente significativo que se debería al tamaño de muestra. Pocos estudios han evaluado esta variable, nuestros resultados confirman los hallazgos en Ferreñafe, Lambayeque en la Costa norte ${ }^{15} \mathrm{y}$ en Polonia ${ }^{27}$, es posible que los machos y las hembras están expuestos en forma similar; sin embargo, las perras en etapa de gestación podrían tener mayor riesgo para infectarse con A. caninum y $T$. canis, la secreción de la prolactina durante esta etapa y la lactancia podrían estimular la reactivación de sus larvas hipobióticas.

La edad fue el único factor asociado. Los perros menores de 1 año tendrían 10 veces más riesgo de adquirir la infección por $T$. canis que los mayores de 1 año, los cachorros por tener más vías de infección (transplacentaria, lactogénica, o ingestión) están en mayor riesgo de infectarse y a tener una mayor carga parasitaria que los adultos ${ }^{2}$. Además, es posible que la mayoría de estos animales son criados en pisos de tierra y el hábito de los perros de reposar en los suelos probablemente contaminados favorece la transmisión por $T$. canis. Se ha sugerido una posible asociación entre la edad y la prevalencia ${ }^{14}$. Los cachorros tienen prevalencias más elevadas por $T$. canis que los adultos en Canberra ${ }^{28}$, en Polonia los animales menores de 3 meses tenían $58,1 \%$ de prevalencia que decreció con la edad alcanzando 2,5\% los mayores de 1 año $^{27}$. En este estudio se intenta identificar algunos factores asociados a la infección por helmintos zoonóticos en perros, este hallazgo tiene importancia para diseñar medidas de prevención y control. Se sugiere realizar estudios sobre factores de riesgo, debido a que los parques públicos de la zona urbana y urbano marginal de $\operatorname{Lima}^{24,29}$ y de la ciudad de Chincha presentan altas tasas de contaminación por huevos de Toxocara sp. ${ }^{18}$. Aunque, los resultados del presente estudio con un diseño transversal respecto a los factores de riesgo no es el más apropiado por el tamaño de muestra, el pequeño número de variables y por que no se ha usado métodos de regresión logística, se sugiere estudios de caso-control o de cohorte para identificar los verdaderos factores de riesgo en la población canina.

\section{RESUMEN}

El objetivo de este trabajo fue determinar la prevalencia de infección por helmintos enteroparásitos del perro e identificar algunos factores asociados en una zona urbana de la ciudad de Ica, al sur del Perú. Se realizó un estudio transversal-descriptivo entre noviembre a diciembre de 2001, en 162 perros con dueño, de ambos sexos, diferentes edades y razas seleccionados por un muestreo bietápico. Se evaluaron dos muestras por animal mediante examen directo y de concentración (Faust y Sedimentación espontánea en tubos de ensayo). Se definió como caso a los animales que resultaron positivos a helmintos al examen coproparasitológico. La prevalencia general fue $40,12 \%$, para Toxocara canis $19,75 \%$, Ancylostoma caninum 9,26\%, Dipylidium caninum 8,64\%, Toxascaris leonina $6,17 \%$ y Taenia sp. 4,32\%. El sexo no está asociado a la infección por helmintos intestinales, la edad menor de un año $\mathrm{p}=0,00000002$, OR 9,74 IC 95\% $(3,75$ 
- 25,72) es el único factor de riesgo potencial hallado para la infección por T. canis.

\section{REFERENCIAS}

1.- SOULSBY E. Parasitología y enfermedades parasitarias en animales domésticos. $7^{\mathrm{a}}$ ed. Interamericana S.A, México DF, 823 pp, 1987.

2.- LEGUIA P G. Enfermedades parasitarias de perros y gatos. Epidemiología y control. $2^{\mathrm{a}} \mathrm{ed}$. Editorial de Mar EIRL, Lima 155 pp 2002.

3.- GEORGI J, GEORGI M. Parasitología en clínica canina. Ed. Interamericana SA, México DF, México 231 pp 1994.

4.- SCHANTZ P, GLICKMAN L. Ascáridos de perros y gatos: Un problema de salud pública y de medicina veterinaria. Bol Of Sanit Panam 1983; 94: 571-85.

5.- ACHA P N, SZYFRES B. Zoonosis y enfermedades trasmisibles comunes al hombre y a los animales. Publicación Científica 503. 2 ${ }^{\mathrm{a}}$ Ed. Washington, DC: Organización Panamericana de la Salud, 1986.

6.- MAGUIÑA C, HERNÁNDEZ H, GOTUZZO E et al. Larva migrans visceral. Primer reporte en el Perú. Rev Méd Herediana 1991; 2: 14-7.

7.- RUBILAR L, ZAPATA L, MORENO G, CERDA S. Prevalencia de Echinococcus granulosus y de otros céstodes de perros en la comuna del Carmen Nuble, Chile. Parasitol al Día 1985; 9: 55-7.

8.- GORMAN T, YÁNEZ V, ALCAÍNO H. Coccidias intestinales en caninos en la comuna de San Miguel, Región Metropolitana, Chile. Rev Av Cs Vet 1989; 4: 57-62.

9.- OLIVEIRA-SEQUEIRA T C G, AMARANTE A F T, FERRARI T B, NUNES C L. Prevalence of intestinal parasites in dogs from São Paulo State, Brazil. Vet Parasitol 2002; 103:19-27.

10.- TORNO O, GARCÍA S, PRAT M, SANTAMA-RÍA B. Enteroparásitos del perro en un sector de Bahía Blanca, Argentina. Parasitol al Día 1996; 20: 144-6.

11.- RODRÍGUEZ-VIVAS R, COB-GALERA L, DOMÍNGUEZ-ALPIZAR J. Frecuencia de pa-rásitos gastrointestinales en animales domésticos diagnosticados en Yucatán, México. Rev Biomed 2001; 12: $19-25$.

12.- JUNCHAYA J. Contribución al estudio del Ancylostoma caninum en perros de ciudad de Lima. Tesis de Bachiller en Medicina Veterinaria, Universidad Nacional Mayor de San Marcos, Lima, Perú. 78 pp 1964.

13.- TRIVEÑO F L I. Estudio de Diphyllobothrium y otros helmintos en humanos, gatos, perros y peces de la ciudad de Lima. Tesis de Bachiller en Medicina Veterinaria, Universidad Nacional Mayor de San Marcos, Lima, Perú. 36 pp 1970.

14.- BAZAN H, CASTILLO Y, SALAZAR R, SAEZ G. Enteroparásitos en Canis familiaris en S.J.L. Libro de Resúmenes del IV Congreso Peruano de Parasitología.
Lima, Perú p 209, 2000.

15.- ALVAREZ SEM. Encuesta sobre diphyllobotriasis canina en la provincia de Ferreñafe. Tesis de Médico Veterinario, Universidad Nacional Pedro Ruíz Gallo. Lambayeque, Perú. 35 pp 1976.

16.- LEGUIA G, GUERRERO C, ARÉVALO F. Prevalencia de helmintos gastrointestinales en caninos de la Provincia de Huancayo. Rev Invest Pecuaria IVITA UNMSM 1980; 5:15-20.

17.- RODRÍGUEZ V, MUÑIZ F. Toxocara canis en excretas de perros, suelos y vegetales de calles, plazas y áreas recreacionales de Cusco urbano. Libro de Resúmenes del IV Congreso Peruano de Para-sitología. Lima, Perú. p 161, 2000.

18.- DÁVAlOS A M, PACHAS G O, PÉREZ E V. Toxocariosis en Canis familiaris y suelo en el Distrito de Chincha Alta (1998-1999). Libro de Resúmenes del IV Congreso Peruano de Parasitología. Lima, Perú. p $152,2000$.

19.- CABRERA M. Prevalencia de echinococosis canina en la zona perimétrica del Camal Municipal de Chincha. 1996-1998. Tesis de Médico Veterinario y Zootecnista, Universidad Nacional San Luis Gonzaga de Ica. Chincha, Perú. 40 pp 1998.

20.- HENDRIX C M. Diagnóstico parasitológico veterinario. $2^{\mathrm{a}}$ ed. Harcourt Brace de España. Madrid, España. 311 pp 1999.

21.- TELLO R, CANALES M. Técnicas de diagnóstico de enfermedades causadas por enteroparásitos. Diagnóstico 2000; 39: 197-8.

22.- ROJAS C M. Manual parasitológico: Morfología, quimioterapia y prevención. Ed. Maijosa. Lima, Perú. 60 pp 1993.

23.- MIRANDA - SOUSA A, ALZAMORA B, MAGUIÑA $C$ et al. Primer reporte en el Perú de toxocariasis ocular: análisis de 21 casos. Bol Soc Per Med Inter 1999; 12: 20-8.

24.- ZEVALLOS L S A, CHIEFFI P P, PERES B A et al. Soil contamination and human infection by Toxocara sp. in the urban area of Lima, Peru. Mem Inst Oswaldo Cruz 1998; 93: 733-4.

25.- LEGUA P, GUERRA J, BUSSALLEU A. Larva migrans cutánea en Lima. Reporte de 3 casos. Rev Méd Herediana 1990; 1: 47-50.

26.- VILLANUEVA R C G, YATACO M E M. Helmintiasis en la Provincia de Chincha - Ica. Libro Resúmen Simposio Internacional: Parasitismo intestinal en el hombre. Lima, Perú. 1988.

27.- LUTY T. Prevalence of species of Toxocara in dogs, cats and red foxes from the Poznan region, Poland. J Helminthol 2001; 75: 153-6.

28.- NICHOLAS W L, KRAYSHEK J. Intestinal helminth parasites in dogs. Austr Vet J 1982; 59: 191.

29.- CASTILLO Y, BAZAN H, ALVARADO D, SAEZ G. Estudio epidemiológico de Toxocara canis en parques recreacionales del Distrito de San Juan de Lurigancho, Lima-Perú. Parasitol al Día 2001; 25: 109-14. 